\title{
Post-intervention Durability of Alcohol Care Management: 1-Year Follow-up of a Randomized Controlled Trial
}

\author{
James R. Rotenberg, $M D^{1,2}$, Shirley H. Leong, $P h D^{7}$, Stephen A. Maisto, $P h D^{3,4}$, \\ James R. McKay, PhD ${ }^{1,2}$, Kyle Possemato, PhD ${ }^{3,4}$, Erin Ingram, $B A^{7}$, and David W. Oslin, $M D^{1,2}$ \\ 'Department of Veterans Affairs, Mental Illness Research, Education, and Clinical Center (MIRECC), Center of Excellence for Substance Abuse \\ Treatment and Evaluation, Corporal Michael J. Crescenz Veterans Affairs Medical Center, Philadelphia, PA, USA; ${ }^{2}$ Department of Psychiatry, \\ University of Pennsylvania Perelman School of Medicine, Philadelphia, PA, USA; ${ }^{3}$ Department of Psychology, Syracuse University, Syracuse, NY, USA; \\ ${ }^{4}$ Department of Veterans Affairs, VA Center for Integrated Healthcare, Syracuse, NY, USA.
}

\author{
KEY WORDS: addiction; primary care; randomized trials; alcoholism; \\ addictive behavior. \\ J Gen Intern Med 33(10): 1626-8 \\ DOI: $10.1007 / \mathrm{s} 11606-018-4567-7$ \\ (C) Society ofGeneral Internal Medicine (This is a U.S. government work and \\ not under copyright protection in the U.S.; foreign copyright protection \\ may apply) 2018
}

\section{INTRODUCTION}

Alcohol care management (ACM) is one model of embedding a behavioral health provider (BHP) into primary care clinics. ${ }^{1,2}$ We previously reported on the effectiveness of a 6-month trial of ACM vs referral to outpatient specialty care (SC) for treatment engagement and reducing alcohol consumption. ${ }^{3}$ Though designed to empower patient/primary care provider (PCP) dyads to independently manage problematic alcohol use, the durability of ACM following the BHP's departure is unknown: Here, we evaluate the post-intervention effects of ACM on drinking outcomes. It was predicted that, compared to SC referral, ACM-mediated improvements in alcohol consumption would persist over the 6 months following intervention.

\section{METHODS}

This was a pre-planned 1-year follow-up analysis of a 26-week single-blind multisite randomized clinical trial of ACM versus referral to SC. The trial is registered with Clinicaltrials.gov (NCT00419315), and the methods were previously described in detail. ${ }^{3}$ Briefly, PCPs referred alcohol-dependent adults to an integrated behavioral health clinic through an existing screening program. Those randomized to ACM met periodically with their assigned BHP during which the BHP provided manualized counseling ("Medical Management,"") and encouraged alcohol reduction and adherence to naltrexone-based pharmacotherapy (PCP-prescribed with support from the BHP). Selfreported drinking was elicited at 9 and 12 months postrandomization using the Time Line Follow-Back. ${ }^{5}$ Primary

Trial Registration Number: NCT00419315 (Clinicaltrials.gov)

Published online July 12, 2018 outcomes were the monthly presence of drinking (both any or heavy), and the percent days drinking (PDD), percent days heavy drinking (PDHD), and drinks per drinking day (DPDD).

Repeated monthly summaries of drinking outcomes were compared using generalized linear models, with logistic and identity link functions for binary and continuous outcomes respectively, fit using generalized estimating equations (GEE). All models included a pre-treatment version of the response as a covariate, together with binary indicators for intervention group (ACM vs SC) and site, and a linear trend for time measured relative to both baseline and 6 months later to account for treatment termination. Group-by-time covariates, site-by-time covariates, and group-by-site interactions were also assessed for inclusion in the models by comparing the Quasi-likelihood Information Criterion (QIC). ${ }^{6}$ The significance of the follow-up time and interaction terms was evaluated based on $p$ values from score tests in the GEE, at a conventional significance level of alpha $=0.05$. For consistency with the original report, ${ }^{3}$ a compound symmetry structure was used for the working correlation matrix, and continuous measures of drinking were analyzed after log transformation.

\section{RESULTS}

Of the 163 participants randomized, 127 provided data on drinking throughout 12 months of follow-up: The 36 lost to follow-up were more likely to be women and have better initial physical functioning (SF-12 Physical score), though adjusting for these covariates yielded similar results (data not shown).

Twenty-nine participants (of 65 for whom data is available) received naltrexone prescriptions in the post-intervention period: 25 and 4 in the ACM and SC groups respectively (average and stdev days into follow-up period 115.12, 58.61 for ACM; 121.25, 78.28 for SC).

Main effects models fit best for most alcohol responses based on the QIC except for the presence of any drinking. There were no significant covariate interactions between treatment and site, treatment and time, or site and time (all $p$ values $>0.07$ ). Only the interaction between treatment and time adjusted at 6 months was marginally significant for $\log (\mathrm{PDD})$ $(p=0.07)$. Despite the main effects model not being the best fit 
Table 1 Change in Trajectory and Treatment Effect in the Follow-up Phase

\begin{tabular}{|c|c|c|c|c|c|c|}
\hline$\overline{\text { Outcome }}$ & OR/Beta & SE & $95 \% \mathrm{CI}$ & Chi-square & DF & $p$ value \\
\hline \multicolumn{7}{|c|}{ Change in trajectory from treatment phase to follow-up phase } \\
\hline Any heavy drinking & 1.05 & 0.06 & $(0.93,1.18)$ & 0.62 & 1 & 0.43 \\
\hline Any drinking & 1.09 & 0.06 & $(0.98,1.21)$ & 2.68 & 1 & 0.10 \\
\hline $\log (\mathrm{PDHD})$ & 0.04 & 0.05 & $(-0.06,0.14)$ & 0.65 & 1 & 0.42 \\
\hline $\log (\mathrm{PDD})$ & 0.04 & 0.04 & $(-0.04,0.13)$ & 1.15 & 1 & 0.28 \\
\hline $\log (\mathrm{DPDD})$ & 0.02 & 0.02 & $(-0.02,0.06)$ & 1.24 & 1 & 0.26 \\
\hline \multicolumn{7}{|c|}{ Treatment effect in the follow-up phase } \\
\hline Any heavy drinking & 0.52 & 0.13 & $(0.32,0.85)$ & 6.80 & 1 & 0.009 \\
\hline Any drinking & 0.78 & 0.24 & $(0.43,1.42)$ & 0.66 & 1 & 0.42 \\
\hline $\log (\mathrm{PDHD})$ & -0.62 & 0.22 & $(-1.05,-0.18)$ & 7.29 & 1 & 0.007 \\
\hline $\log (\mathrm{PDD})$ & -0.21 & 0.24 & $(-0.68,0.26)$ & 0.76 & 1 & 0.38 \\
\hline $\log (\mathrm{DPDD})$ & -0.19 & 0.12 & $(-0.42,0.04)$ & 2.60 & 1 & 0.11 \\
\hline
\end{tabular}

PDHD, percent days heavy drinking; PDD, percent days drinking; DPDD, drinks per drinking day

for the presence of any drinking, all interaction terms were non-significant $(p>0.17)$.

There was no indication of moderation of treatment effect post-intervention. During the follow-up, as compared to treatment period, there were no statistically significant differences in the rates of change for any of the five primary drinking outcomes examined (see Table 1). There remained a significant effect of ACM on prevalence of heavy drinking $\left(\chi^{2}(1)=\right.$ $6.80, p=0.01$ ) with fewer heavy drinkers in ACM than SC $(\mathrm{OR}=0.52,95 \% \mathrm{CI}=(0.32,0.85))$. PDHD also favored ACM (see Fig. 1). Other outcomes were not statistically significantly different between the ACM and SC groups (see Table 1).

\section{DISCUSSION}

We have demonstrated that the benefits of an alcohol intervention integrated into primary care and emphasizing naltrexone pharmacotherapy persist, unreduced, for the 6 months following treatment. ACM implementation should be studied in future work. Results suggest that a focus on pharmacotherapy in the context of a supportive integrated primary care team may have sustained effects.

Contributors: Kevin G. Lynch PhD (Department of Psychiatry, University of Pennsylvania Perelman School of Medicine);

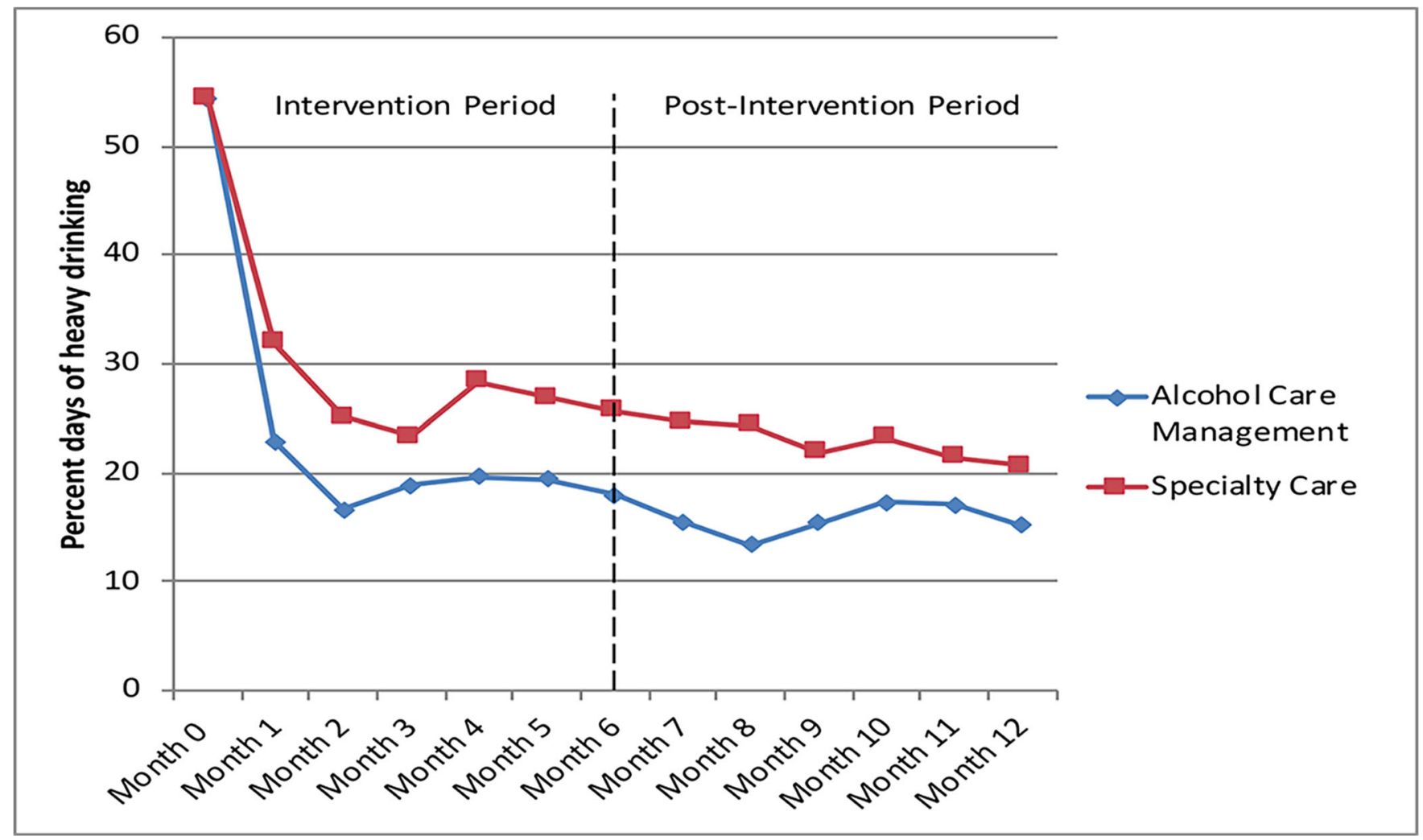

Fig. 1 Group means of the percent days of heavy drinking during intervention and post-intervention periods. The vertical dashed line represents the end of the intervention. 
Larry J. Lantinga, PhD (Department of Psychology, Syracuse University; and VA Center for Integrated Healthcare, Department of Veterans Affairs, Syracuse, NY)

Corresponding Author: David W. Oslin, MD; Department of Veterans Affairs, Mental Illness Research, Education, and Clinical Center (MIRECC), Center of Excellence for Substance Abuse Treatment and Evaluation, Corporal Michael J. Crescenz Veterans Affairs Medical Center, Philadelphia, PA, USA (e-mail: Dave.Oslin@va.gov).

Funders Support for the design and conduct of the study, and collection, management, analysis, and interpretation of the data was provided by the Department of Veterans Affairs Health Services Research and Development Program (Dr. Oslin, "Primary Care Based Disease Management for Alcohol Dependence" HSRD IIR 06-058); and the VISN 4 Mental Illness Research, Education, and Clinical Center at the Philadelphia VA Medical Center.

Writing of this manuscript was supported by the Office of Academic Affiliations, Advanced Fellowship Program in Mental Illness Research and Treatment, Department of Veterans Affairs (Dr. Rotenberg).

\section{Compliance with ethical standards:}

Prior presentations: There have been no prior presentations of the currently discussed follow-up outcome data.
Conflict of interest: Dr. Leong discloses a spouse who is employed by the pharmaceutical company Bristol Meyers Squibb. Dr. McKay discloses consulting for the biotechnology company Triggr Health. All other authors declare no conflicts of interest.

\section{REFERENCES}

1. Willenbring ML, Olson DH. A randomized trial of integrated outpatient treatment for medically ill alcoholic men. Arch Intern Med. 1999; 159(16): 1946-52.

2. Oslin DW, Klaus J, Ingram E et al. Foundations for integrated care: Behavioral solutions for primary care. Department of Veterans Affairs. 2013. Available at: https://www.mirecc.va.gov/visn4/BHL/BHLresources3.asp. Accessed 7 July 2017.

3. Oslin DW, Lynch KG, Maisto SA et al. A randomized clinical trial of alcohol care management delivered in department of veterans affairs primary care clinics versus specialty addiction treatment. $\mathrm{J}$ Gen Intern Med. 2014;29(1):162-8.

4. Pettinati HM, Mattson ME. Medical management treatment manual: A clinical guide for researchers and clinicians providing pharmacotherapy for alcohol dependence. (generic version). National Institute on Alcohol Abuse and Alcoholism. 2010. Available at: https://pubs.niaaa.nih.gov/publications/MedicalManual/MMManual.pdf. Accessed 7 July 2017.

5. Sobell LC, Sobell MB, Leo GI, Cancilla A. Reliability of a timeline method: Assessing normal drinkers' reports of recent drinking and a comparative evaluation across several populations. Br J Addict. 1988;83(4):393-402.

6. Pan W. Akaike's information criterion in generalized estimating equations. Biometrics. 2001:57(1):120-5. 